\title{
LOCALIZACIÓN DE LA INDUSTRIA AGROALIMENTARIA EN ARAGÓN ${ }^{1}$
}

\author{
E. Climent López y E. Ruiz Budría \\ Universidad de Zaragoza \\ ecliment@unizar.es; eruiz@unizar.es
}

\begin{abstract}
Resumen: La industria agroalimentaria evoluciona en dos direcciones contrapuestas, la de los productos estandarizados o globales y la de los productos especializados o locales, bifurcación que puede explicarse por medio de la teoría de los mundos de producción. A la luz de dicha teoría se estudia la localización de la industria agroalimentaria en Aragón, constatándose la existencia de dos modelos: la producción de alimentos globales se concentra en la aglomeración urbano-industrial de Zaragoza, mientras que la de alimentos locales se localiza en una serie de áreas rurales concretas, ligada a producciones agrarias fuertemente arraigadas en el territorio.
\end{abstract}

Palabras clave: Industria agroalimentaria, mundos de producción, localización, Aragón.

\begin{abstract}
Food industry is bifurcating into two zones of production: that of standardized or global foods, on one hand, that of specialized or local foods, on the other hand. This bifurcation can be explained by means of the worlds of production theory. On the light of this theory, location of food industry in Aragon (Spain) is studied. Two location patterns have been identified: global food industries are concentrated within the urban-industrial agglomeration of Zaragoza, while local food production is located within specific rural areas linked to agricultural productions strongly embedded to territory.
\end{abstract}

Keywords: Food industry, worlds of production, location, Aragón (Spain).

Recibido: 3-12-10. Aceptado: 3-11-11.

${ }^{1} \mathrm{El}$ artículo presenta resultados del proyecto de investigación "Gobernanza, innovación y convenciones en las comarcas vitivinícolas de Aragón: tipología y prospectiva de Denominaciones de Origen a partir de la teoría de los mundos de producción" (CSO2008-05793-CO3-02), financiado por el Ministerio de Ciencia e Innovación. 


\section{Introducción}

Desde los años setenta del pasado siglo se asiste a la estandarización creciente del sistema agroalimentario, tanto en los procesos de producción agrícolas e industriales como en los hábitos de consumo; dicho proceso tiene sus principales protagonistas en las empresas multinacionales del sector y en las grandes superficies de distribución, que han fomentado pautas estandarizadas de producción en serie y de consumo de masas, respectivamente. Pero, junto a dicha tendencia, se observa también la emergencia de un sistema agroalimentario local alternativo, basado en prácticas de producción y consumo no estandarizadas (Ilbery, 2001, p. 6). Dicho de otra manera, el sector de la alimentación se está bifurcando en dos tipos principales: primero, el de las redes de alimentos globales, estandarizados e industrializados; segundo, el de los procesos de producción especializada (Murdoch y Miele 1999, p. 469). En el primero la competencia se establece en precio, mientras que en el segundo se establece en diferenciación y calidad. Este último responde a dos tendencias clave: por una parte, la emergencia en los países capitalistas de consumidores que no se conforman con los productos alimentarios estandarizados y demandan mayor variedad y diversidad; por otra parte, la búsqueda de alimentos más saludables y seguros, en respuesta a las crisis alimentarias recientes asociadas a los procesos industriales, como, por ejemplo, la "enfermedad de las vacas locas".

La producción estandarizada encaja bien en los supuestos de la economía neoclásica, pero la especializada, basada en la calidad y la diferenciación, se resiste a hacerlo. Para introducir este segundo tipo en el análisis económico se ha recurrido a la teoría de las convenciones, formulada en sus líneas generales por Boltansky y Thévenot (1991). Las convenciones se definen como prácticas, rutinas y acuerdos, informales o institucionalizados, que permiten, entre otras cosas, identificar la calidad de los productos. Aplicando dicha teoría a la industria Salais y Storper (1992) distinguen cuatro tipos ideales de "mundos de producción", que definen como combinaciones coherentes de tecnologías, mercados, calidades de los productos y prácticas cuantitativas de uso de recursos. Cada mundo de producción puede entenderse como un conjunto de convenciones que expresan los consensos forjados a lo largo de la cadena de producción respecto a la conducta que cabe esperar de cada actor y las calidades que debe tener un producto (Sánchez, Aparicio y Alonso, 2010, p. 470).

Los cuatro mundos de producción se establecen atendiendo a dos dimensiones de los productos: primero, la tecnología de producción, distinguiéndose si es estandarizada o especializada; segundo, el tipo de mercado al que se dirigen, distinguiéndose si es genérico o dedicado. Así, se identifican: primero, el mundo de producción industrial, que es el de los productos estandarizados y genéricos; segundo, el mundo del mercado en red, que es el de los productos estandarizados y dedicados; tercero, el mundo marshalliano o de los productos especializados y dedicados, y finalmente el 
mundo de la innovación o de los productos genéricos y especializados (Salais y Storper, 1992, p. 172).

Aplicando la teoría de los mundos de producción a la dualidad o bifurcación observada en la industria agroalimentaria, Murdoch y Miele (1999), Murdoch, Marsden y Banks (2000) identifican las redes de alimentos globales, estandarizados e industrializados con el mundo de producción industrial y los procesos de producción especializada con los mundos de producción marshalliano y de la innovación.

La teoría de los mundos de producción tiene implicaciones espaciales en las que, sin embargo, la bibliografía apenas ha incidido. Por lo que respecta a la industria agroalimentaria, los productos que encajan en el mundo de producción industrial son calificados como "globales" (Murdoch y Miele 1999, p. 469) y los que corresponden a los mundos de producción marshalliano y de la innovación como "locales" (Ilbery, 2001, p. 6). Los términos en que se expresa dicha contraposición sugieren que las industrias respectivas podrían verse afectadas por factores de localización diferentes: las de alimentos globales seguirían las pautas generales de la industria, disponiendo de una gran movilidad, mientras que las de alimentos locales mostrarían un fuerte arraigo respecto a determinados lugares; en este sentido, no es casual que, para referirse al mundo de producción de productos especializados y dedicados, Salais y Storper hayan utilizado la denominación de marshalliano, en clara referencia a la teoría del distrito industrial.

En la misma línea argumental, las industrias de productos estandarizados se asocian con procesos de deslocalización: "desde los años setenta la estandarización de los procesos de producción agro-alimentaria y de las pautas de consumo masivo ha fomentado una mundialización, con su consiguiente ‘deslocalización', de los sistemas de producción alimentaria" (Ilbery, 2001, p. 6); por el contrario, el desarrollo de las industrias de alimentos locales se asocia con procesos de relocalización: "hay un genuino interés en la relocalización de los alimentos y el desarrollo de cadenas de suministro alimentarias alternativas en territorios específicos, especialmente los que han quedado marginados por los procesos de globalización" (Ilbery et al., 2002, p. 257).

En síntesis, las industrias agroalimentarias de productos estandarizados y genéricos, siguiendo las pautas esperables en el mundo de producción industrial, serían industrias globales, no arraigadas y prestas a moverse en busca de localizaciones en las que maximizar los beneficios. Como estas industrias compiten básicamente en precio, buscarán emplazamientos donde puedan reducir al máximo los costes de producción (laborales, de suelo) y de transporte. Por el contrario, las industrias de productos especializados, siguiendo las pautas esperables en los mundos de producción marshalliano y de la innovación, serían industrias locales, fuertemente arraigadas en 
determinados territorios. Como estas industrias compiten en diversificación y calidad, su emplazamiento estará fuertemente ligado al medio natural, que condiciona la producción de las materias primas, y al saber hacer o tradición específica.

\section{Objetivos y metodología}

El objetivo que se persigue es analizar la localización de la industria agroalimentaria aragonesa a la luz de la teoría de los mundos de producción. Además, teniendo en cuenta la escasa incidencia de la bibliografía sobre las implicaciones espaciales de dicha teoría, se espera que el análisis aporte elementos de juicio útiles para afinar la contraposición entre industrias globales y locales y mejorar el conocimiento sobre sus pautas de localización respectivas.

Aragón dispone de una base agraria amplia y diversificada, que constituye una buena fuente de aprovisionamiento de materias primas para la industria agroalimentaria. La estructura territorial de la región se caracteriza por un marcado contraste entre la aglomeración urbano-industrial de Zaragoza, una de las mayores de España, y el resto del territorio, de baja densidad demográfica y carácter rural, articulado por villas y pequeñas ciudades. Lo que resulta esperable en dichas circunstancias es que las industrias agroalimentarias locales se ubiquen en determinados ámbitos rurales, de acuerdo con su producción agraria y el saber hacer acumulado a lo largo de la historia y que las industrias globales se localicen en torno a Zaragoza, donde mejor pueden minimizar los costes de transporte.

El problema metodológico inicial consiste en determinar cuáles son las industrias globales y cuáles las locales. Las estadísticas oficiales se ajustan a la Clasificación Nacional de Actividades Económicas (CNAE-93), de carácter jerárquico, cuyas categorías pueden servir de base para una primera aproximación. La industria agroalimentaria comprende todo el grupo 15, que se divide en 9 subgrupos identificados mediante 3 dígitos (del 151 al 159); alguno de éstos, como el 157 (fabricación de productos para la alimentación animal), puede incluirse en su totalidad en el mundo de producción industrial, pues elabora productos estandarizados y genéricos, pero en otros casos surgen dudas cuya resolución exige bajar de nivel jerárquico. Así, por ejemplo, dentro del subgrupo 159 (elaboración de bebidas) la actividad 1592 (destilación de alcohol etílico procedente de fermentación) puede incluirse en el mundo de producción industrial, mientras que la actividad 1593 (elaboración de vinos) encaja mejor en el mundo marshalliano, pues hoy en día se orienta mayoritariamente a la elaboración de productos especializados y dedicados. Bajar hasta el nivel siguiente de la CNAE permite afinar mejor, pero no puede perderse de vista que quienes siguen 
las convenciones correspondientes a cada mundo de producción son las empresas y éstas pueden seguir orientaciones diferentes dentro de un mismo nivel de la CNAE, como muestran Sánchez, Aparicio y Alonso (2010) en relación con la industria del vino; más aún, hay empresas que elaboran productos de diferentes mundos de producción, como han puesto de manifiesto Murdoch y Miele (1999).

Identificar las industrias globales y locales empresa por empresa exigiría el diseño y elaboración de una encuesta de amplio alcance, tarea no abordable en las circunstancias actuales. Por ello se ha optado por clasificar a priori como globales o locales las diferentes actividades de la industria agroalimentaria y, a partir de ahí, recurrir a las fuentes estadísticas disponibles para obtener la información pertinente. El criterio de clasificación elegido se basa en un indicio seguro, aunque estricto: en el marco de la Unión Europea las industrias de productos locales ponen de manifiesto su carácter local recurriendo a la certificación, si bien no todas las certificaciones (labels) acreditan un origen local. Ilbery et al. (2002, p. 259) distinguen tres tipos de esquemas de certificación, según que se basen en el producto, el proceso o el lugar: el primero promueve productos particulares elaborados dentro de un área determinada de acuerdo con técnicas prescritas; el segundo promueve determinados procesos de producción o transformación; el tercero promueve productos originarios de un territorio particular. El primero y el último son los que responden claramente a la idea de alimentos locales, pues el segundo certifica un proceso, sin que importe el lugar concreto de producción, como ocurre en el caso de los alimentos ecológicos.

En consecuencia se han considerado industrias locales aquéllas que tienen establecido un sistema de certificación basado en el producto o en el lugar: en el caso de Aragón dichas industrias son las del jamón (denominación de origen "Jamón de Teruel"), el aceite de oliva (denominaciones de origen "Aceite del Bajo Aragón" y "Aceite Sierra del Moncayo"), el queso (varias certificaciones de artesanía agroalimentaria y calidad alimentaria) y el vino (cuatro denominaciones de origen y seis zonas de vino de la tierra). No toda la producción de estos alimentos o bebidas tiene la certificación correspondiente, pero el cotejo de los directorios de industrias agroalimentarias y de las páginas Web de las denominaciones de origen y demás indicaciones geográficas muestra que la mayor parte de las empresas de los primeros están incluidas en las segundas, lo cual es un buen indicador del carácter local de dichas actividades. Las demás industrias se consideran globales.

Una vez establecida dicha clasificación se ha recurrido, como fuente de información al Censo de Industrias del Gobierno de Aragón del año 2001, que es el último realizado. Dicho censo incluye información detallada de todas las empresas. Se han seleccionado los datos relativos a la actividad industrial, el tamaño y la localización. La actividad aparece especificada hasta el nivel de 5 dígitos de la CNAE, lo que permite diferenciar las industrias locales de las globales; de entre los indicadores de 
tamaño se ha elegido el número de trabajadores y respecto a la localización se han tomado las comarcas como marco espacial de referencia.

\section{La localización de la industria agroalimentaria en Aragón}

Según dicho censo, la industria agroalimentaria ocupa a 15.622 trabajadores, cifra equivalente al 14 por ciento del total de la industria aragonesa. Al nivel de dos dígitos de la CNAE es la segunda rama en cuanto a empleo, siendo superada sólo por la de material de transporte. Presenta un alto grado de concentración espacial en la comarca de Zaragoza, donde se localizan 6.457 trabajadores, el 41 por ciento del total (tabla 1). Otro 31 por ciento se distribuye entre siete comarcas, cada una de las cuales aporta entre 500 y 1.000 empleos, es decir, entre el 3 y el 6 por ciento del total, cifras muy alejadas de las de Zaragoza. Una primera constatación puede realizarse ya: la industria agroalimentaria en Aragón adopta unas pautas de localización similares a las del resto de la industria, mostrando una clara preferencia por la aglomeración urbana de Zaragoza. No obstante, el grado de concentración es menor que el del conjunto, pues la comarca de la capital concentra el 53 por ciento del empleo total de la industria.

La identificada como industria local, según el criterio antes explicitado, genera 1.630 empleos, que equivalen al 10 por ciento de la industria agroalimentaria. En este grupo destaca claramente la elaboración de vino, con el 47 por ciento de dichos empleos; le siguen el jamón y el aceite de oliva, con el 27 y el 23, respectivamente, y finalmente el queso, con un testimonial 3 por ciento.

La localización de esta industria local se aleja notablemente del modelo general de la industria agroalimentaria: en la comarca de Zaragoza se localizan 83 empleos, es decir, un modesto 5 por ciento, siendo claramente superada por otras siete comarcas, que concentran entre todas el 74 por ciento, oscilando sus valores individuales entre el 15 y el 6. Se trata de Campo de Cariñena, Somontano de Barbastro, Bajo Aragón, Valdejalón, Comunidad de Calatayud, Teruel y Campo de Borja (tabla 1 y figura 1). Las 25 comarcas restantes presentan unos valores de empleo netamente inferiores a los citados, llegando a ser nulos o meramente testimoniales en la mayoría de ellas. El modelo es claramente distinto del general, puesto que no hay una fuerte concentración en Zaragoza; pero se constata que estas industrias presentan también un alto grado de selectividad espacial, puesto que no se da una amplia dispersión por todo el territorio regional.

Analizando caso por caso, la industria del vino concentra el 94 por ciento del empleo en cinco comarcas, las que albergan a las cuatro denominaciones de origen 
(Campo de Cariñena, Somontano de Barbastro, Comunidad de Calatayud y Campo de Borja) más una de las que incluyen una indicación geográfica de vinos de la tierra (Valdejalón). El 60 por ciento del empleo de la industria del jamón se concentra en tres comarcas de la provincia de Teruel: la de la capital, Bajo Aragón y GúdarJavalambre. Por lo que respecta al aceite de oliva, el 77 por ciento de sus trabajadores se concentra en 7 comarcas, ocupando el primer lugar Bajo Aragón, (denominación de origen del mismo nombre), con el 26 por ciento; las restantes son: Hoya de Huesca, Campo de Borja (denominación de origen Aceite del Moncayo), Bajo Martín, Somontano de Barbastro, Matarraña y Caspe. La producción de quesos, mucho menos importante en términos cuantitativos de empleo, presenta un modelo muy próximo al general, pues el 32 por ciento del mismo se concentra en la comarca de Zaragoza.

\section{Tipología agroalimentaria de las comarcas aragonesas}

Atendiendo a la importancia de la industria agroalimentaria y al grado de especialización en industrias locales, cabe clasificar las comarcas aragonesas (excluida la de Zaragoza) en los grupos siguientes:

1) Comarcas con una industria agroalimentaria potente y fuertemente especializada en industrias locales: lo integran Campo de Cariñena, Somontano de Barbastro, Bajo Aragón y Valdejalón. El volumen de empleo total de su industria agroalimentaria se encuentra entre 300 y 600 trabajadores, estando fuertemente especializadas en las industrias locales, con coeficientes comprendidos entre 4,4 y 5,5 (tabla 1). Cada una de ellas aporta entre el 10 y el 15 por ciento de los trabajadores de las industrias locales de Aragón y conjuntamente el 50 por ciento, lo que les otorga el papel más relevante dentro de éstas en la región. Bajo Aragón presenta una cierta diversificación (aceite de oliva y jamón), mientras que las demás están especializadas en el vino.

2) Comarcas con una industria agroalimentaria potente y escasa presencia de industrias locales: lo integran Hoya de Huesca, Cinco Villas, La Litera, Jiloca y Bajo Cinca. El empleo de su industria agroalimentaria oscila entre 300 y 1.000 trabajadores, pero sus coeficientes de especialización en industrias locales son muy bajos (entre 0,07 y 0,45), aportando poco más del 4 por ciento del empleo total de Aragón. La industria agroalimentaria de estas comarcas es de carácter global.

3) Comarcas con una industria agroalimentaria potente y especializadas en industrias locales: lo integran Comunidad de Calatayud, Teruel y Campo de Borja. El número de trabajadores de su industria agroalimentaria oscila entre $300 \mathrm{y}$ 1.000 y su coeficiente de especialización en industrias locales entre 1,7 y 3,1. 
Tabla 1. Distribución por comarcas del empleo de la industria agroalimentaria en Aragón.

\begin{tabular}{|l|c|c|c|c|c|c|c|}
\hline Comarca & Total & Jamón & $\begin{array}{c}\text { Aceite } \\
\text { de } \\
\text { oliva }\end{array}$ & Queso & Vino & Local & $\begin{array}{c}\text { Coeficiente } \\
\text { de especiali- } \\
\text { zación }\end{array}$ \\
\hline Zaragoza & 6.457 & 46 & 9 & 14 & 14 & 83 & 0,12 \\
Hoya de Huesca & 917 & 3 & 40 & 0 & 0 & 43 & 0,45 \\
Comunidad de Calatayud & 915 & 10 & 13 & 0 & 139 & 162 & 1,70 \\
Cinco Villas & 738 & 0 & 1 & 5 & 0 & 6 & 0,08 \\
La Litera & 618 & 0 & 8 & 0 & 0 & 8 & 0,12 \\
Jiloca & 585 & 3 & 0 & 0 & 4 & 7 & 0,11 \\
Bajo Cinca & 536 & 0 & 4 & 0 & 0 & 4 & 0,07 \\
Teruel & 508 & 123 & 0 & 5 & 0 & 128 & 2,41 \\
Campo de Cariñena & 422 & 8 & 0 & 0 & 234 & 242 & 5,50 \\
Bajo Aragón & 413 & 81 & 99 & 9 & 1 & 190 & 4,41 \\
Somontano de Barbastro & 407 & 27 & 31 & 0 & 155 & 213 & 5,02 \\
Valdejalón & 357 & 18 & 16 & 0 & 136 & 170 & 4,56 \\
Campo de Borja & 308 & 0 & 36 & 0 & 63 & 99 & 3,08 \\
Cinca Medio & 260 & 0 & 2 & 0 & 0 & 2 & 0,07 \\
Los Monegros & 259 & 0 & 4 & 0 & 0 & 4 & 0,15 \\
Bajo Aragón - Caspe & 250 & 0 & 25 & 0 & 8 & 33 & 1,27 \\
Ribera Alta del Ebro & 244 & 0 & 0 & 0 & 0 & 0 & 0,00 \\
Tarazona y el Moncayo & 216 & 0 & 15 & 1 & 0 & 16 & 0,71 \\
Gúdar-Javalambre & 166 & 57 & 0 & 0 & 0 & 57 & 3,29 \\
Matarraña & 154 & 23 & 29 & 1 & 9 & 62 & 3,86 \\
Campo de Daroca & 147 & 0 & 0 & 0 & 4 & 4 & 0,26 \\
Ribera Baja del Ebro & 142 & 0 & 0 & 0 & 0 & 0 & 0,00 \\
La Jacetania & 137 & 0 & 0 & 0 & 0 & 0 & 0,00 \\
La Ribagorza & 131 & 0 & 0 & 0 & 0 & 0 & 0,00 \\
Bajo Martín & 81 & 0 & 32 & 5 & 0 & 37 & 4,38 \\
Cuencas Mineras & 55 & 23 & 0 & 2 & 2 & 27 & 4,70 \\
Andorra-Sierra de Arco & 42 & 0 & 4 & 0 & 0 & 4 & 0,91 \\
Sierra de Albarracín & 39 & 0 & 0 & 0 & 0 & 0 & 0,00 \\
Alto Gállego & 35 & 0 & 0 & 0 & 0 & 0 & 0,00 \\
Campo de Belchite & 24 & 0 & 11 & 0 & 3 & 14 & 5,59 \\
Sobrarbe & 22 & 0 & 0 & 0 & 0 & 0 & 0,00 \\
Maestrazgo & 21 & 11 & 0 & 2 & 0 & 13 & 5,93 \\
Aranda & 16 & 0 & 0 & 0 & 2 & 2 & 1,20 \\
ARAGÓN & & & & & & & 1,00 \\
\hline
\end{tabular}

* Para obtener el coeficiente de especialización primero se dividen los empleos locales y totales de cada comarca y de Aragón; luego se divide el cociente obtenido para cada comarca entre el obtenido para Aragón.

Fuente: Gobierno de Aragón: Departamento de Industria, Comercio y Desarrollo (2001). 


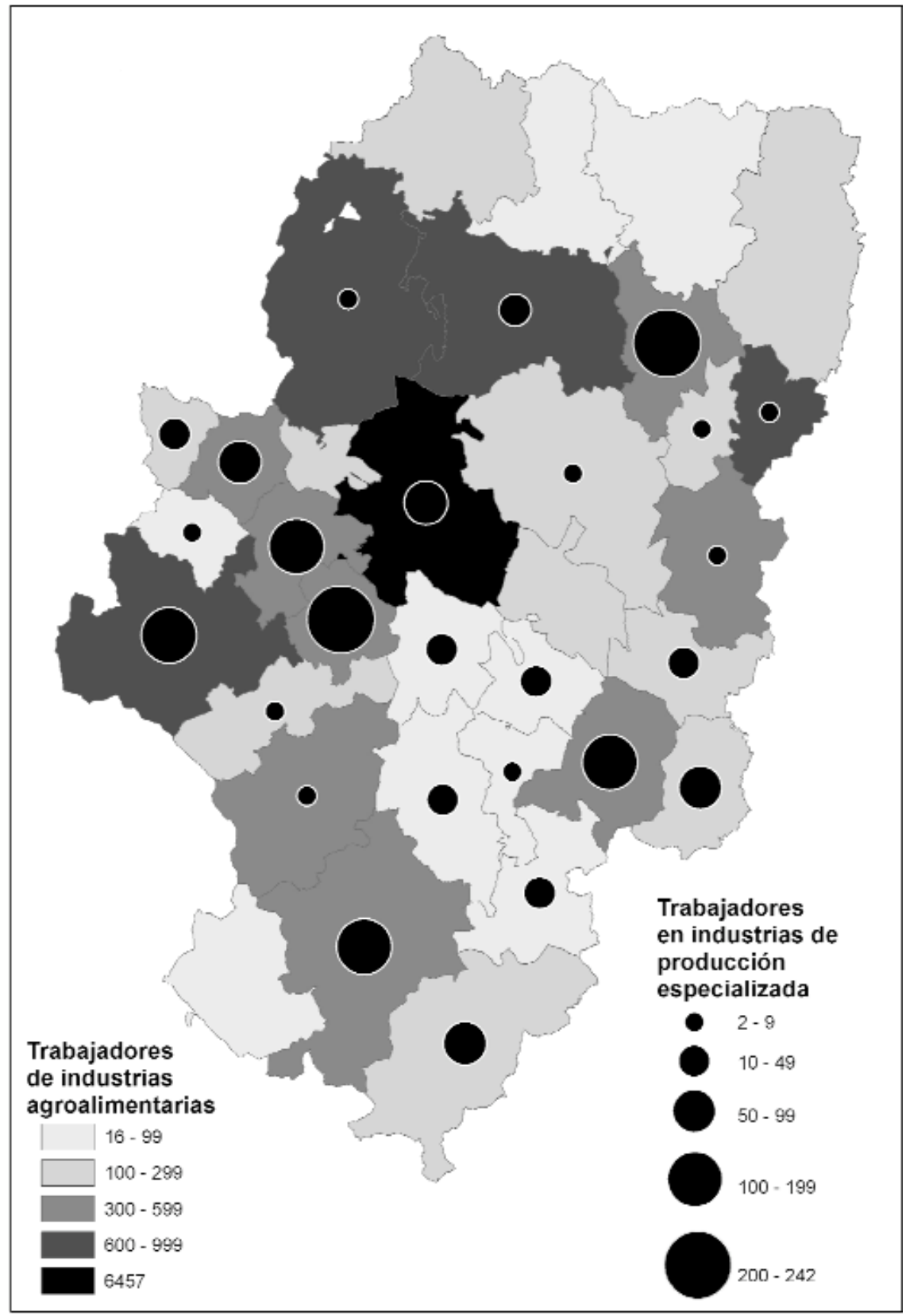

Figura 1: Distribución espacial de la industria agroalimentaria.

Fuente: Gobierno de Aragón: Departamento de Industria, Comercio y Desarrollo (2001). 
Aportan individualmente entre el 6 y el 10 por ciento del empleo de la industria local y conjuntamente el 24; la de Teruel destaca especialmente en la industria del jamón y las otras dos en la del vino, a la que acompaña en Campo de Borja la del aceite de oliva. Las tres comarcas, no obstante, cuentan con un significativo volumen de empleo en industrias agroalimentarias globales, presentando rasgos intermedios entre los dos grupos anteriores.

4) Comarcas con escasa industria agroalimentaria, pero fuerte especialización en industrias locales: lo integran Gúdar-Javalambre, Matarraña, Bajo Martín, Cuencas Mineras y Maestrazgo, todas ellas de la provincia de Teruel. Los empleos de la industria agroalimentaria varían entre 20 y 200, pero sus coeficientes de especialización en industrias locales están comprendidos entre 3 y 6, aportando conjuntamente el 12 por ciento del empleo de éstas. En todas ellas destaca claramente la industria del jamón, excepto en Bajo Martín, donde destaca la del aceite de oliva y en Matarraña, donde ambas comparten protagonismo.

5) Comarcas con escasa industria agroalimentaria y muy escasa presencia o ausencia total de industrias locales. Lo integran las 15 restantes, la mitad de las comarcas aragonesas.

\section{Conclusiones y consideraciones finales}

En el trabajo se ha constatado que en Aragón se da la bifurcación del sector agroalimentario entre industrias globales y locales. El peso de las primeras es sensiblemente mayor que el de las segundas: 90 y 10 por ciento, respectivamente, si bien estas cifras hay que tomarlas con cierta cautela, dado que el criterio utilizado para identificar las industrias locales es muy estricto. Las seleccionadas como tal (vino, jamón, aceite de oliva y queso) en el caso de Aragón, salvo contadas excepciones, responden claramente a lo que se ha denominado industrias locales. Pero se tiene constancia de que dentro de los restantes grupos de la industria agroalimentaria hay empresas que trabajan en la misma línea, si bien no es posible identificarlas a partir del uso exclusivo de las fuentes estadísticas disponibles; la aplicación de un procedimiento más afinado muy probablemente elevaría la proporción de las industrias locales.

La hipótesis de partida puede considerarse confirmada: primero, porque se ha constatado que las industrias globales y locales siguen pautas de localización diferentes. Segundo, porque las industrias globales muestran un elevado grado de concentración en la aglomeración urbana de Zaragoza, siguiendo las pautas generales de 
localización del sector: el conjunto de la industria agroalimentaria concentra en la comarca de la capital el 41 por ciento de sus empleos, pero en la global la proporción sube al 45, cifra que se aproxima mucho al 53 del conjunto del sector industrial. Sin duda alguna es en Zaragoza donde mejor se pueden minimizar los costes de transporte, lo cual es importante para unas industrias que compiten en precio.

Las industrias locales, por el contrario, se localizan en ámbitos rurales concretos, poniendo así de manifiesto un arraigo particular respecto a las áreas en que las condiciones naturales y una larga tradición garantizan la obtención de una materia prima diferenciada o el dominio de un proceso de elaboración específico. Conviene destacar que no se trata de industrias dispersas por todo el territorio de la región, sino que muestran un alto grado de selectividad espacial, lo cual permite asociarlas claramente con el mundo de producción marshalliano.

Ha podido comprobarse también la existencia de comarcas que tienen un cierto peso en las industrias globales e incluso en éstas y las locales a la vez, aunque a mucha distancia respecto a Zaragoza en las primeras. Ello es coherente con el hecho, ya mencionado, de que la industria agroalimentaria presenta un grado de concentración en la aglomeración urbana inferior a la media del sector industrial. Es la constatación de que este grupo o rama de la industria tiene mayor propensión que otras a localizarse en el medio rural, como ponen de manifiesto diversos trabajos, como el de Borobio y Frutos (1986), que estudian el caso aragonés. El medio rural ofrece la oportunidad de minimizar algunos costes de producción (mano de obra y suelo, fundamentalmente), junto a la ventaja de la proximidad a las materias primas; no obstante, dicha propensión está muy condicionada por la posibilidad de minimizar también los costes de transporte, por lo que la industria agroalimentaria muestra una clara selectividad especial hacia las áreas bien situadas respecto a las infraestructuras viarias, como es el caso de las comarcas incluidas en los grupos 2 y 3 del apartado anterior.

Como consideración final cabe añadir que LEADER, la iniciativa política de referencia para el desarrollo rural en la Unión Europea, teniendo como filosofía de partida la orientación de abajo-arriba, se ha centrado en suscitar iniciativas de desarrollo endógenas que nazcan y se desarrollen a partir de un fuerte arraigo en el territorio (Massam y Esparcia, 1998). La promoción de las industrias locales es un buen camino para ello, por lo que las políticas e iniciativas de desarrollo rural más interesantes son las encaminadas a potenciar la producción de alimentos especializados y dedicados. La instalación en el medio rural de industrias para la transformación de los productos agrarios es más fácil y duradera cuando se trata de estos alimentos que de los estandarizados y genéricos. Algunas noticias recientes parecen abonar dicha idea: así, mientras que la industria de elaboración del vino se desarrolla notablemente y no cesa de crecer en las comarcas tradicionales, como Campo de Cariñena (Loscertales, 
2009), la comarca del Jiloca se enfrenta al cierre de un gran matadero, porque las economías de escala propias de una industria típica del mundo de producción industrial se pueden conseguir mejor en otros emplazamientos.

\section{Bibliografía}

Boltansky, L. y Thévenot, L. (1991). De la justification. Les économies de la grandeur. Paris, Gallimard.

Borobio, M.P. y Frutos Mejías, L.M. (1986) Algunos aspectos de la agroindustria en Aragón. El sistema agrario aragonés: perspectivas y problemas. Zaragoza, Institución Fernando el Católico, pp. 95-118.

Ilbery, B. (2001). Relocalización de los sistemas de producción agroalimentaria en la Unión Europea. Geographicalia 39, pp. 5 20.

Ilbery, B., Kneafsey, M., Maye, D. Buller, H., y Morris, C. (2002). Labels with(out) a cause?. A comparative analysis of product labelling schemes in North America and Europe. Innovation in rural areas. 4 colloque franco-britannique de géographie rurale pp. 255-273.

Loscertales Palomar, B. (2009). La Denominación de Origen Cariñena: una apuesta por la singularidad y la calidad en un mundo globalizado. Zaragoza, Ediciones 94.
Massam, B.H. y Esparcia, J.P. (1998). Approaches to the LEADER program of the European Union: a conceptual overview. Toronto, York University.

Murdoch, J. y Miele, M. (1999). 'Back to Nature': changing 'worlds of production' in the food sector. Sociologia Ruralis 39, pp. $465-483$.

Murdoch, J., Marsden, T. y Banks, J. (2000). Quality, nature and embeddedness: some theoretical considerations in the context of the food sector. Economic Geography 76, pp. $107-125$.

Salais, R. y Storper, M. (1992). The four 'worlds' of contemporary industry. Cambridge Journal of Economics 16, pp. 169-193.

Sánchez-Hernández, J.L., Aparicio-Amador, J., Alonso-Santos, J.L. (2010). The shift between worlds of production as an innovative process in the wine industry in Castile and Leon (Spain). Geoforum 41, pp. $469-478$ 